\title{
T1 Recovery Is Predominantly Found in Black Holes and Is Associated with Clinical Improvement in Patients with Multiple Sclerosis
}

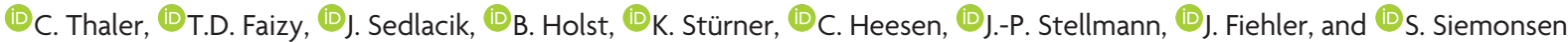

\begin{abstract}
BACKGROUND AND PURPOSE: Quantitative MR imaging parameters help to evaluate disease progression in multiple sclerosis and increase correlation with clinical disability. We therefore hypothesized that T7 values might be a marker for ongoing tissue damage or even remyelination and may help increase clinical correlation.
\end{abstract}

MATERIALS AND METHODS: MR imaging was performed in 17 patients with relapsing-remitting MS at baseline and after 12 months of starting immunotherapy with dimethyl fumarate. On baseline images, lesion segmentation was performed for normal-appearing white matter, T2 hyperintense (FLAIR lesions), T1 hypointense (black holes), and contrast-enhancing lesions, and T1 relaxation times were obtained at baseline and after 12 months. Changes in clinical status were assessed by using the Expanded Disability Status Scale and Symbol Digit Modalities Test at both dates (Expanded Disability Status Scale-difference/Symbol Digit Modalities Test-diff).

RESULTS: The highest T1 relaxation time at baseline was measured in black holes (1460.2 $\pm 209.46 \mathrm{~ms})$ followed by FLAIR lesions (1400.38 \pm $189.1 \mathrm{~ms})$, pure FLAIR lesions (1327.5 $\pm 210.04 \mathrm{~ms})$, contrast-enhancing lesions (1205.59 $\pm 199.95 \mathrm{~ms})$, and normal-appearing white matter (851.34 $\pm 30.61 \mathrm{~ms})$. After 12 months, 71 values had decreased significantly in black holes (1369.4 $\pm 267.81 \mathrm{~ms})$, contrast-enhancing lesions $(1079.57 \pm 183.36 \mathrm{~ms})$ (both $P<.001)$, and normal-appearing white matter $(841.98 \pm 36.1 \mathrm{~ms}, P=.006)$. With the Jonckheere-Terpstra Test, better clinical scores were associated with decreasing $T 1$ relaxation times in black holes $(P<.05)$.

CONCLUSIONS: T1 relaxation time is a useful quantitative MR imaging technique, which helps detect changes in MS lesions with time. We assume that these changes are associated with the degree of myelination within the lesions themselves and are pronounced in black holes. Additionally, decreasing $\mathrm{Tl}$ values in black holes were associated with clinical improvement.

ABBREVIATIONS: $\mathrm{BH}=$ black hole; $\mathrm{CE}-\mathrm{L}=$ contrast-enhancing lesion; EDSS = Expanded Disability Status Scale; NAWM = normal-appearing white matter; MP2RAGE = double inversion-contrast magnetization-prepared rapid acquistion of gradient echo; SDMT = Symbol Digit Modalities Test; T1-RT = T1 relaxation time

$\mathbf{M}_{\mathrm{r}}^{\mathrm{R}}$ $\mathrm{R}$ imaging is an established tool in diagnosing multiple sclerosis and in monitoring inflammatory disease progression. In clinical routine, $\mathrm{T} 2$ and $\mathrm{T} 1$ lesion load and the detection of contrast-enhancing lesions (CE-Ls) are commonly used for monitoring subclinical disease activity and evaluating the effectiveness of pharmaceutical treatments. While hyperintense lesions on T2weighted images (FLAIR lesions) correspond to a wide spectrum of histopathologic changes, ranging from edema and mild demy-

Received June 16, 2016; accepted after revision September 8.

From the Departments of Diagnostic and Interventional Neuroradiology (C.T. T.D.F., J.S., B.H., J.F., S.S.) and Neurology (K.S., C.H., J.-P.S.) and Institute for Neuroimmunology and Clinical MS Research (K.S., C.H., J.-P.S., S.S.), University Medical Centre Hamburg-Eppendorf, Hamburg, Germany.

Please address correspondence to Christian Thaler, MD, University Medical Centre Hamburg-Eppendorf, Martinistr 52, 20246 Hamburg-Eppendorf, Germany; e-mail: c.thaler@uke.de

三 Indicates article with supplemental on-line tables.

http://dx.doi.org/10.3174/ajnr.A5004 elination to glial scars or liquid necrosis, nonenhancing T1 hypointense lesions, black holes (BHs), are reported to be more specific markers for demyelination, axonal loss, and tissue damage. ${ }^{1-6}$ It was observed that for discriminating the different stages of cell damage, the degree of $\mathrm{BH}$ hypointensity seems to reflect the extent of axonal loss and might distinguish demyelinated and partially remyelinated lesions. ${ }^{7,8}$ Consequently, $\mathrm{T} 1$ relaxation times (T1RTs) are increased in edema, demyelination, and axonal loss. ${ }^{9-11}$

A recent study showed that the assessment of MS lesions by their $\mathrm{T} 1$ values helps to increase correlations with disability and might lead to a more differentiated lesion classification. ${ }^{12}$ However, little is known about the potential of lesional $\mathrm{T} 1$ values as a clinical marker in disease progression. Only a small number of previous studies have applied T1 relaxometry in patients with MS and reported increased $\mathrm{T} 1$ values in normal-appearing white matter (NAWM). ${ }^{13,14}$ Therefore, our study focuses on the longitudinal evaluation of T1 values in different MS lesion types, representing different grades of tissue destruction. With the recently introduced 


\section{Subject population}

\begin{tabular}{lccc}
\hline & Mean & SD & Range \\
\hline Age (yr) & 35.1 & 9.5 & $18-51$ \\
EDSS baseline & 2.2 & 1.6 & $0-6.5$ \\
Disease duration $(\mathrm{yr})$ & 7.7 & 6.1 & $0.1-19$ \\
Sex & 11 women, 6 men & & \\
Disease course & 17 RRMS & & \\
\hline
\end{tabular}

Note:-RRMS indicates relapsing-remitting MS.

double inversion-contrast magnetization-prepared rapid acquisition of gradient echo (MP2RAGE) sequences, it is now possible to generate quantitative $\mathrm{T} 1$ maps with high reproducibility. ${ }^{15}$

The purpose of this study was to observe the evolution of $\mathrm{T} 1$ values in NAWM and in lesions in patients with MS for 1 year after starting immunotherapy with dimethyl fumarate. We hypothesized that longitudinal changes of lesional T1 values are associated with changes in clinical disability because T1 values might be a marker for ongoing tissue damage or even remyelination.

\section{MATERIALS AND METHODS}

MR imaging was performed in 17 patients with MS at baseline and after 12 months. Also, at both time points, the clinical status was assessed by experienced neurologists. For this study, all patients who decided to be treated with dimethyl fumarate were treated at the MS day hospital. The study was approved by the local Ethical Committee Hamburg (Ethik-Kommission der Ärztekammer Hamburg) following the guidelines of the Declaration of Helsinki, and patients provided written informed consent. At baseline, all patients were diagnosed with relapsing-remitting MS, of whom 1 transitioned to secondary-progressive MS at follow-up. Disease duration ranged from 1 month to 19 years. All patients started treatment with dimethyl fumarate after baseline scans. For further patient characteristics, see the Table.

\section{MR Imaging Data Acquisition}

All MR imaging at baseline and after 12 months was performed on a 3T scanner (Magnetom Skyra; Siemens, Erlangen, Germany). The MR imaging protocol included a sagittal 3D FLAIR sequence $(\mathrm{TE}=390 \mathrm{~ms}, \mathrm{TR}=4700 \mathrm{~ms}, \mathrm{TI}=1800 \mathrm{~ms}, 192$ sections, $\mathrm{FOV}=$ $256 \mathrm{~mm}$, voxel size $=1.0 \times 1.0 \times 1.0 \mathrm{~mm}$ ), a T1-weighted magnetization-prepared rapid acquisition of gradient echo sequence before and after gadolinium injection $(\mathrm{TE}=2.43 \mathrm{~ms}, \mathrm{TR}=1900$ $\mathrm{ms}, \mathrm{TI}=900 \mathrm{~ms}, 192$ sections, $\mathrm{FOV}=256 \mathrm{~mm}$, voxel size $=1.0 \times$ $1.0 \times 1.0 \mathrm{~mm}$, flip-angle $=9^{\circ}$ ), and a double inversion-contrast magnetization-prepared rapid acquisition of gradient echo sequence before contrast agent injection $(\mathrm{TE}=2.98 \mathrm{~ms}, \mathrm{TR}=5000$ $\mathrm{ms}, \mathrm{TI}=700 \mathrm{~ms}, 176$ sections, $\mathrm{FOV}=256 \mathrm{~mm}$, voxel size $=1.0 \times$ $1.0 \times 1.0 \mathrm{~mm}$, flip angle $=4^{\circ}$ ).

\section{Image Analysis}

For measuring T1-RT in NAWM, 6 ROIs were manually defined in the occipital, frontal, and parietal white matter on baseline and follow-up MP2RAGE images. Simultaneously, ROI placement was controlled in the corresponding FLAIR images to avoid partial volume errors from MS lesions.

In baseline scans, FLAIR lesions were semiautomatically segmented on FLAIR images by using an open-source lesionsegmentation software (Lesion Segmentation Tool for SPM;
http://www.academia.edu/2729347/LST_A_Lesion_Segmentation_ Tool_For_SPM). ${ }^{16}$ For optimal lesion segmentation, the final threshold was set at $\kappa=0.3$. If necessary, lesion outlines were corrected manually. FLAIR images were linearly registered to the MP2RAGE images on baseline and follow-up images, and the corresponding transformation was applied to the FLAIR lesion masks.

Additionally, BH lesion segmentation was performed by 2 independent raters with a minimum of 1-year specific training in MS image diagnostics and evaluation on T1-weighted MPRAGE images. "BHs" were defined as nonenhancing lesions that appear hypointense on T1WI with signal intensity below the cortex and are concordant with hyperintense lesions on a T2WI. Lesions were outlined by using the software Analyze 11.0 (AnalyzeDirect, Overland Park, Kansas). The BH lesion masks of both raters were binarized and multiplied patient-wise to obtain a consensus mask. Finally, the consensus masks were registered to MP2RAGE images at baseline and follow-up. We marked CE-Ls, applying the same algorithm as described for $\mathrm{BH}$ lesion outlining and registered them to nonenhanced MP2RAGE images.

Finally, we calculated a pure FLAIR lesion mask by subtracting the $\mathrm{BH}$ and CE-L mask from the corresponding FLAIR lesion mask, leaving only those lesions that were visible on FLAIR but did not correspond to a BH or CE-L.

An example of lesional ROI placement is shown in Fig 1. Due to the registration of the baseline and follow-up images, we understand that minor displacements of lesions ROIs might occur. To minimize this possible bias, we excluded lesions of $<5$ voxels and assessed lesion maps on follow-up images to exclude misplaced ROIs and correct them if necessary. For each patient, for FLAIR lesions, BHs, pure FLAIR lesions, and CE-L number, volumes were calculated, and lesion-wise, median $\mathrm{T} 1$ values were obtained at baseline and follow-up. New FLAIR lesions and CE-Ls were detected manually after 12 months and were listed separately.

Because larger lesions might have a greater impact on clinical disability, we adapted the changes in T1-RTs to the actual lesion size in which it was measured. To correct for lesion size, we calculated a weighted average of the lesional T1-RTs (ie, T1-RTs were multiplied by their lesion volume and afterward divided by the patient's total lesion volume).

\section{Clinical Status}

At baseline and follow-up, clinical status was assessed by experienced neurologists by using the Expanded Disability Status Scale (EDSS) according to published guidelines. ${ }^{17}$ In addition, the oral Symbol Digit Modalities Test (SDMT) was assessed, and an SD for an age- and education-matched control cohort was calculated. ${ }^{18}$

\section{Statistical Analysis}

Statistical analysis was performed by using R 3.0.0 statistical and computing software (http://www.r-project.org/) and SPSS 21.0 (IBM, Armonk, New York). A paired $t$ test was used to compare lesion T1-RTs at baseline and after 12 months within lesion groups. The Jonckheere-Terpstra Test (a rank-based nonparametric test) was used to test for changes in T1-RTs in patient groups classified by clinical changes. ${ }^{19}$ Two-sided $P$ values $<.05$ were statistically significant.

AJNR Am J Neuroradiol 38:264-69 Feb 2017 www.ajnr.org 


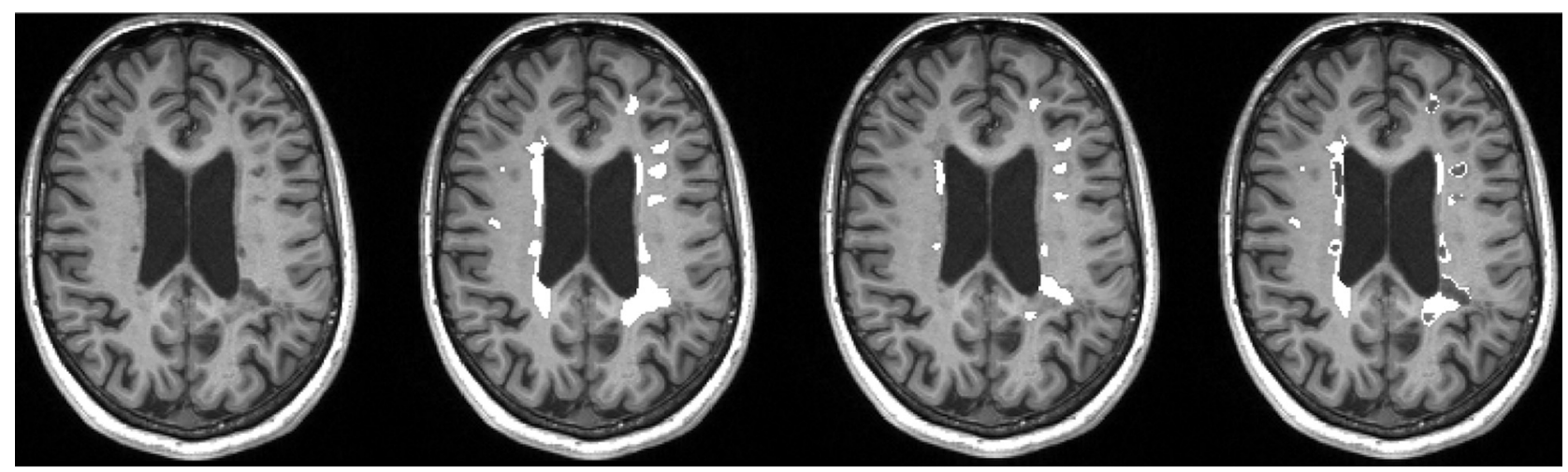

FIG 1. An example of ROI placement in TIWI MPRAGE at baseline. From left to right: 1) TIWI MPRAGE transversal without lesion ROIs, 2) ROIs indicating FLAIR-lesion masks, 3) ROls indicating black hole lesion masks, and 4) ROls indicating FLAIR-lesion masks without black holes (pure FLAIR lesion masks).

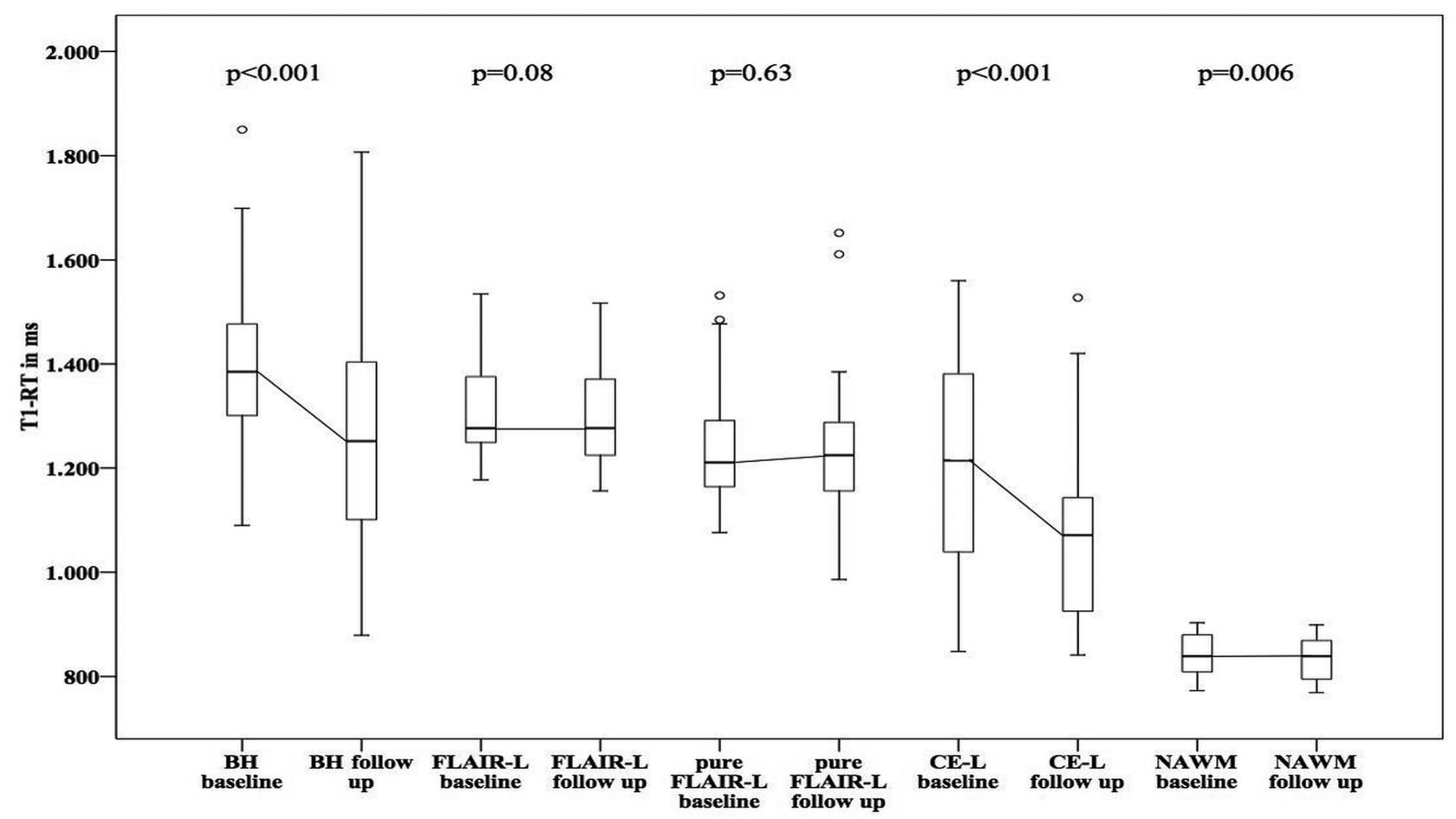

FIG 2. Boxplots showing $\mathrm{T} 1$ relaxation times at baseline and after 12-month follow-up for different lesion types. $P$ values were calculated by using a paired $t$ test.

\section{RESULTS}

\section{MR Imaging Measurements}

At baseline, at least 1 FLAIR lesion was detected in all patients, and $\mathrm{BHs}$ were detected in all except 1 patient. Overall, 380 FLAIR lesions, $504 \mathrm{BHs}$, and 362 pure FLAIR lesions were segmented on baseline scans. In 8 of 17 patients, 22 CE-Ls were detected at baseline. After 12 months, 1 patient demonstrated a new CE-L and 7 patients presented with new FLAIR lesions. Interrater reliability for the detection of black holes was high, with a Dice coefficient of 0.916 .

T1 values in NAWM showed a significant decrease with T1RTs at a baseline of $851.34 \pm 30.61 \mathrm{~ms}$ to $841.98 \pm 36.1 \mathrm{~ms}$ after 12 months $(P=.006)$. In MS lesions, the highest T1-RT at baseline was measured in BHs (1460.21 $\pm 209.46 \mathrm{~ms})$ followed by T1-RTs in FLAIR lesions (1400.38 $\pm 189.1 \mathrm{~ms})$ and T1-RTs in pure FLAIR lesions (1327.5 $\pm 210.04 \mathrm{~ms}$ ). In 8 of 17 patients, 22
CE-Ls were detected at baseline, with a mean T1-RT of $1205.59 \pm$ 199.95 ms. After 12 months, T1 values decreased in all lesion types: $\mathrm{BHs}=1369.4 \pm 267.81 \mathrm{~ms}$, FLAIR lesions $=1383.33 \pm$ $274.53 \mathrm{~ms}$, pure FLAIR lesions $=1322.64 \pm 305.07 \mathrm{~ms}$, CE-Ls $=$ $1079.57 \pm 183.36 \mathrm{~ms}$. Changes in T1-RT were calculated for all lesion ROIs. Using paired $t$ tests, we found significant differences only for T1-RTs measured in NAWM $(P=.006)$, BHs, and CE-Ls (both $P<.001)$ at baseline and after 12 months, but not for FLAIR lesions $(P=.08)$ or pure FLAIR lesions $(P=.63)$. A detailed overview of mean T1-RTs in FLAIR lesions, BHs, pure FLAIR lesions, and CE-Ls at baseline and follow-up is given in Fig 2 and On-line Tables 1 and 2.

There were no significant associations for changes in T1-RTs in $\mathrm{BHs}$ and NAWM or any other lesion type. 

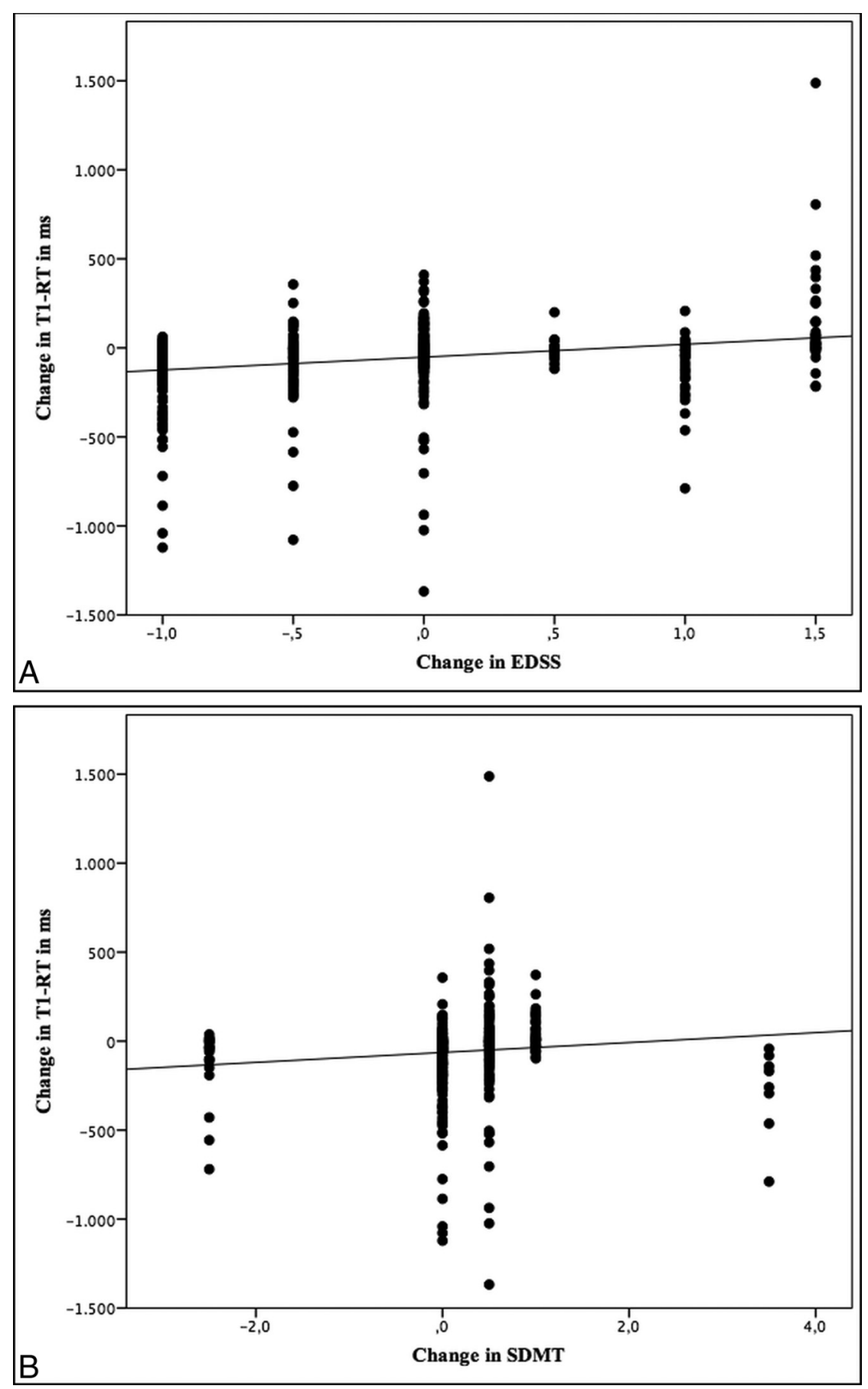

FIG 3. Scatterplots showing an association between lesional changes in $\mathrm{T}$ relaxation times and clinical scores.

\section{Clinical Measurements}

EDSS scores were obtained for all patients at baseline and followup. The mean EDSS score at baseline was 2.21 and changed only marginally to 2.18 after 12 months. In a subset of 14 patients, the SDMT was available. A mean SDMT-SD at baseline of -0.07 slightly increased to 0.21 after 12 months. With the JonckheereTerpstra Test, better clinical scores in EDSS and SDMT were associated with decreasing T1-RTs in BHs $(P<.001)$ (Fig 3). However, there were no significant differences for changes in T1-RTs in NAWM, FLAIR lesions, pure FLAIR lesions, or CE-Ls.

\section{DISCUSSION}

For monitoring disease progression and the effectiveness of pharmaceutical treatment in MS, the number of T2, T1, and contrast-enhancing lesions presents a relevant diagnostic tool in clinical routine. However, with routinely used semiquantitative MS imaging biomarkers such as $\mathrm{T} 1$ and $\mathrm{T} 2$ lesion load or the number of FLAIR lesions and CE-Ls, clinical-radiologic correlations are still limited because MS lesions have histopathologic diversity. ${ }^{20-22}$ Therefore, nonconventional MR imaging measures such as T1-RT have shown some promising results in crosssectional and longitudinal studies to improve correlations with disability in patients with MS. ${ }^{12,13,23,24}$ The purpose of this study was to present T1-RT changes in MS lesions for 12 months and, secondly, to show the association of T1-RT changes with clinical disability.

At baseline the highest lesional T1 values were detected in $\mathrm{BHs}$, while the lowest values were measured in CE-Ls; this finding confirms the findings of Blystad et al (2016). ${ }^{25}$ Due to severe tissue destruction, demyelination, and an increase in free water, T1-RTs are expected to be elevated in BHs. In contrast, CE-Ls represent an active inflammation process, associated with an accumulation of inflammatory cells, resulting in lower T1-RTs compared with FLAIR lesions or BHs. ${ }^{26}$

T1-RT alterations were observed in all lesion types and NAWM after 12 months compared with baseline. While T1-RTs in NAWM, BHs, and CE-Ls significantly decreased across time, FLAIR lesions and pure FLAIR lesions did not show meaningful changes of T1-RTs in 12-month follow-up. Our observations are in line with those in former studies, which reported increasing signal intensities of MS lesions in T1WI (comparable with decreasing T1-RTs) in up to $74 \%$ of initially T1WI hypointense lesions. ${ }^{27-29}$ Although we did not evaluate the relative signal intensity, we observed decreasing T1-RTs in $66 \%$ of all $\mathrm{BHs}$, in $82 \%$ of all CE-Ls, and in 54\% of pure FLAIR lesions. However, little is known about decreasing T1-RTs in MS lesions. Bitsch et al $(2001)^{5}$ and Barkhof et al $(2003)^{7}$ demonstrated in histopathologic studies that the intensity of an MS lesion, and therefore its T1-RT, depends, to some extent, on the degree of its myelination. While the intensity of the lesion was decreased in demyelinated lesions, it showed an increase in remyelinated lesions. Remyelination is described as a frequent phenomenon in MS and can be 
extensive in subsets of patients with MS. ${ }^{7,30-33}$ We assume that decreasing T1-RTs in MS lesions depend, to some extent, on the level of remyelination in the lesion itself and might therefore indicate neuronal recovery.

Certainly, other pathologic processes such as resolution of edema, inflammation, or microstructural changes may influence T1-RT as well and might be the main reason for decreasing T1RTs in CE-Ls. However, why overall T1-RTs are stable in pure FLAIR lesions but decrease in BHs remains unclear. One possible explanation could be that BHs represent a bygone, inactive inflammatory process with extensive tissue damage and prevailing neuronal recovery, whereas pure FLAIR lesions still have the potential for ongoing demyelination. This assumption is supported by histopathologic findings from Jonkman et al (2015), ${ }^{34}$ who observed lower T1-RTs in preactive and active lesions than in chronic inactive lesions, classifying pure FLAIR lesions with smaller T1-RTs as active lesions with ongoing demyelination and $\mathrm{BHs}$ with higher T1-RTs as rather chronic inactive lesions.

In this study, we found a significant association between improving clinical status and decreasing T1 values in BHs. So far, studies focusing on changes of T1-RTs in MS lesions and their impact on clinical status are lacking. Manfredonia et al (2007) ${ }^{14}$ observed $\mathrm{T} 1$ values in normal-appearing white and gray matter during a 2-year follow-up in patients with primary-progressive MS and found a significant correlation between increasing T1RTs and clinical impairment. However, Manfredonia et al did not evaluate T1-RTs within MS lesions and included only patients with primary-progressive MS, which might explain the significant increase in T1-RTs, because progressive subtypes of MS are associated with greater damage to white matter. Also, all individuals in our study cohort were treated with dimethyl fumarate, which significantly reduces disease activity as measured by MR imaging in patients with relapsing-remitting $\mathrm{MS}^{35,36}$ and might be a reasonable explanation for decreasing T1 values in NAWM and BHs in our study cohort.

As mentioned before, we hypothesized that changes in T1-RT at least partially represent some changes in the degree of myelination. In contrast to Manfredonia et al, ${ }^{14}$ we predominantly observed decreasing T1 values in NAWM and BHs. However, it is still unclear whether remyelination leads to functional recovery in humans because there is no approved method to measure the degree of demyelination/remyelination in vivo. Yet, promising results were reported in experimental animal models, demonstrating an association between remyelination and functional recovery. ${ }^{37-39}$ To support our findings and especially to confirm an association of lesion evolution and clinical status and to gain deeper insight into the underlying histopathologic process, additional longitudinal studies, including measurements of other quantitative MR imaging parameters such as diffusion tensor imaging, magnetization transfer ratio, and myelin water imaging, are needed.

There was no significant association between the appearance of new FLAIR lesions after 12 months and changes in clinical status. However, when one assesses new lesions in MS, their strategic location is an important feature when correlating with clinical changes. ${ }^{13,40,41}$ It was not the purpose of this study to evaluate the influence of single lesion locations on clinical disability but rather to search for new strategies to assess lesion quality and evolution. Nevertheless, further investigations of the localization of lesions are needed for a better understanding of their clinical impact.

To generate T1 maps, we acquired MP2RAGE sequences, which create a homogeneous T1-weighted contrast with an intrinsic correction of $\mathrm{B} 1$ inhomogeneities and reduce residual proton density and $\mathrm{T} 2{ }^{\star}$ weighting. T1 values measured in MP2RAGE sequences are highly reproducible both across subjects and within the same subject by using different scanning parameters; this feature makes these sequences applicable to longitudinal studies. ${ }^{15}$

\section{CONCLUSIONS}

T1-RT is a useful quantitative MR imaging technique that helps detect changes in MS lesions with time. We assume that these changes are associated with remyelination in the lesions themselves and are predominantly found in BHs rather than in FLAIR lesions or pure FLAIR lesions. Supporting this hypothesis, changes in T1-RT were associated with clinical status, possibly indicating neuronal and even functional recovery. To confirm our findings, further emphasis should be on combining T1-RT with other nonconventional MR imaging techniques such as magnetization transfer ratio, DTI, and myelin water imaging to gain further knowledge of myelin measurement in vivo. Undoubtedly, histopathologic studies would provide the best evidence to verify these MR imaging techniques but are naturally limited.

Disclosures: Christian Thaler-UNRELATED: Support for Travel to Meetings for the Study or Other Purposes: Deutsche Gesellschaft für Neuroradiologie (German Society of Neuroradiology), Comments: travel grant to present study results at the Annual Meeting of the American Society of Neuroradiology in 2016 in Washington, DC. Christoph Heesen-UNRELATED: Grant: German Ministry of Education and Research, Comments: Analysis was part of a Phase II study on boswellic acids in MS.* Jan-Patrick Stellmann_UNRELATED: Consultancy: Genzyme; Grants/Grants Pending: Biogen, Genzyme*; Payment for Lectures including Service on Speakers Bureaus: Biogen, Genzyme; Travel/Accommodations/Meeting Expenses Unrelated to Activities Listed: Novartis. *Money paid to the institution.

\section{REFERENCES}

1. MacKay A, Laule C, Vavasour I, et al. Insights into brain microstructure from the T2 distribution. Magn Reson Imaging 2006;24:515-25 CrossRef Medline

2. Laule C, Leung E, Lis DK, et al. Myelin water imaging in multiple sclerosis: quantitative correlations with histopathology. Mult Scler 2006;12:747-53 CrossRef Medline

3. De Groot CJ, Bergers E, Kamphorst W, et al. Post-mortem MRIguided sampling of multiple sclerosis brain lesions: increased yield of active demyelinating and (p)reactive lesions. Brain 2001;124: 1635-45 CrossRef Medline

4. van Walderveen MA, Kamphorst W, Scheltens P. Histopathologic correlate of hypointense lesions on T1-weighted spin-echo MRI in multiple sclerosis. Neurology 1998;50:1282-88 CrossRef Medline

5. Bitsch A, Kuhlmann T, Stadelmann C, et al. A longitudinal MRI study of histopathologically defined hypointense multiple sclerosis lesions. Ann Neurol 2001;49:793-96 CrossRef Medline

6. Faizy TD, Thaler C, Kumar D, et al. Heterogeneity of multiple sclerosis lesions in multislice myelin water imaging. PLoS One 2016;11: e0151496 CrossRef Medline

7. Barkhof F, Bruck W, De Groot CJ, et al. Remyelinated lesions in multiple sclerosis: magnetic resonance image appearance. Arch Neurol 2003;60:1073-81 CrossRef Medline

8. Riva M, Ikonomidou VN, Ostuni JJ, et al. Tissue-specific imaging is a robust methodology to differentiate in vivo $\mathrm{T} 1$ black holes with 
advanced multiple sclerosis-induced damage. AJNR Am J Neuroradiol 2009;30:1394-401 CrossRef Medline

9. Naruse S, Horikawa Y, Tanaka C, et al. Proton nuclear magnetic resonance studies on brain edema. J Neurosurg 1982;56:747-52 CrossRef Medline

10. Schmierer K, Scaravilli F, Altmann DR, et al. Magnetization transfer ratio and myelin in postmortem multiple sclerosis brain. Ann $\mathrm{Neu}$ rol 2004;56:407-15 CrossRef Medline

11. Schmierer K, Tozer DJ, Scaravilli F, et al. Quantitative magnetization transfer imaging in postmortem multiple sclerosis brain. J Magn Reson Imaging 2007;26:41-51 CrossRef Medline

12. Thaler C, Faizy T, Sedlacik J, et al. T1-thresholds in black holes increase clinical-radiological correlation in multiple sclerosis patients. PLoS One 2015 10:e0144693 CrossRef Medline

13. Parry A, Clare S, Jenkinson M, et al. MRI brain T1 relaxation time changes in MS patients increase over time in both the white matter and the cortex. J Neuroimaging 2003;13:234-39 CrossRef Medline

14. Manfredonia F, Ciccarelli O, Khaleeli Z, et al. Normal-appearing brain $\mathrm{T} 1$ relaxation time predicts disability in early primary progressive multiple sclerosis. Arch Neurol 2007;64:411-15 CrossRef Medline

15. Marques JP, Kober T, Krueger G, et al. MP2RAGE, a self bias-field corrected sequence for improved segmentation and T1-mapping at high field. Neuroimage 2010;49:1271-81 CrossRef Medline

16. Schmidt P, Gaser C, Arsic M, et al. An automated tool for detection of FLAIR-hyperintense white-matter lesions in multiple sclerosis. Neuroimage 2012;59:3774-83 CrossRef Medline

17. Kappos L, Lechner-Scott J, Lienert C. Neurostatus. Neurostatus training and documentation DVD for a standardized neurological examination and assessment of Kurtzke's functional systems and expanded disability status scale for MS patients. 2002, 2007, Basel, Switzerland. http://neurostatus.net; 2015. Accessed January 6, 2015

18. Smith A. The symbol-digit modalities test: a neuropsychologic test of learning and other cerebral disorders. In Helmuth J, ed. Learning Disorders. Seattle: Special Child Publications; 1968;83-91

19. Jonckheere AR. A distribution-free K-sample test against ordered alternatives. Biometrika 1954;41:133-45 CrossRef

20. Miller DH, Altmann DR, Chard DT. Advances in imaging to support the development of novel therapies for multiple sclerosis. Clin Pharmacol Ther 2012;91:621-34 CrossRef Medline

21. Galego O, Gouveia A, Batista S, et al. Brain atrophy and physical disability in primary progressive multiple sclerosis: a volumetric study. Neuroradiol J 2015;28:354-58 CrossRef Medline

22. McFarland HF, Barkhof F, Antel J, et al. The role of MRI as a surrogate outcome measure in multiple sclerosis. Mult Scler 2002;8:40-51 CrossRef Medline

23. Steenwijk MD, Vrenken H, Jonkman LE, et al. High-resolution T1relaxation time mapping displays subtle, clinically relevant, gray matter damage in long-standing multiple sclerosis. Mult Scler 2016; 22:1279-88 CrossRef Medline

24. Simioni $\mathrm{S}$, Amarù F, Bonnier G, et al. MP2RAGE provides new clinically-compatible correlates of mild cognitive deficits in relapsingremitting multiple sclerosis. J Neurol 2014;261:1606-13 CrossRef Medline

25. Blystad I, Håkansson I, Tisell A, et al. Quantitative MRI for analysis of active multiple sclerosis lesions without gadolinium-based contrast agent. AJNR Am J Neuroradiol 2016;37:94-100 CrossRef Medline

26. Filippi M, Rocca MA, Barkhof F, et al; Attendees of the Correlation between Pathological MRI Findings in MS workshop. Association between pathological and MRI findings in multiple sclerosis. Lancet Neurol 2012;11:349-60 CrossRef Medline

27. Bagnato F, Jeffries N, Richert ND, et al. Evolution of T1 black holes in patients with multiple sclerosis images monthly for 4 years. Brain 2003;126:1782-89 CrossRef Medline

28. van Waesberghe JH, van Walderveen MA, Castelijns JA, et al. Patterns of lesion development in multiple sclerosis: longitudinal observations with $\mathrm{T} 1$-weighted spin-echo and magnetization transfer MR. AJNR Am J Neuroradiol 1998;19:675-83 Medline

29. Rovira A, Alonso J, Cucurella G, et al. Evolution of multiple sclerosis lesions on serial contrast-enhanced T1-weighted and magnetization-transfer MR images. AJNR Am J Neuroradiol 1999;20:1939-45 Medline

30. Raine CS, Wu E. Mutlitple sclerosis: remyelination in acute lesions. J Neuropathol Exp Neurol 1993;52:199-204 CrossRef Medline

31. Lassmann W, Brück W, Luchinetti C, et al. Remyelination in multiple sclerosis. Mult Scler 1997;3:133-36 CrossRef Medline

32. Patrikios P, Stadelmann C, Kutzelnigg A, et al. Remyelination is extensive in a subset of multiple sclerosis patients. Brain 2006;129: 3165-72 CrossRef Medline

33. Fox RJ, Cronin T, Lin J, et al. Measuring myelin repair and axonal loss with diffusion tensor imaging. AJNR Am J Neuroradiol 2011;32: 85-91 CrossRef Medline

34. Jonkman LE, Soriano AL, Amor S, et al. Can MS lesion stages be distinguished with MRI? A postmortem MRI and histopathology study. J Neurol 2015;262:1074-80 CrossRef Medline

35. Gold R, Kappos L, Arnold DL, et al; DEFINE Study Investigators. Placebo-controlled phase 3 study of oral BG-12 for relapsing multiple sclerosis. N Engl J Med 2012;367:1098-107 CrossRef Medline

36. Bar-Or A, Gold R, Kappos L, et al. Clinical efficacy of BG-12 (dimethyl fumarate) in patients with relapsing-remitting multiple sclerosis: subgroup analyses of the DEFINE study. J Neurol 2013; 260:2297-305 CrossRef Medline

37. Duncan ID, Brower A, Kondo Y, et al. Extensive remyelination of the CNS leads to functional recovery. Proc Natl Acad Sci U S A 2009;106: 6832-36 CrossRef Medline

38. Liebetanz D, Merkler D. Effects of commissural de- and remyelination on motor skill behaviour in the cuprizone mouse model of multiple sclerosis. Exp Neurol 2006;202:217-24 CrossRef Medline

39. Murray PD, McGavern DB, Sathornsumetee S, et al. Spontaneous remyelination following extensive demyelination is associated with improved neurological function in a viral model of multiple sclerosis. Brain 2001;124(pt 7):1403-16 CrossRef Medline

40. Roosendaal SD, Moraal B, Pouwels PJ, et al. Accumulation of cortical lesions in MS: relation with cognitive impairment. Mult Scler 2009; 15:708-14 CrossRef Medline

41. Rovaris M, Filippi M, Minicucci L, et al. Cortical/subcortical disease burden and cognitive impairment in patients with multiple sclerosis. AJNR Am J Neuroradiol 2000;21:402-08 Medline 